\title{
METRIZABILITY OF GENERAL ANR
}

\author{
KÔICHI TSUDA \\ Dedicated to Professor Keiô Nagami on the occasion of his 60 th birthday
}

\begin{abstract}
We show that every nonmetrizable $\operatorname{ANR}(\mathscr{P})$ contains a copy of a Tychonoff cube of uncountable weight. Hence, every finite dimensional $\operatorname{ANR}(\mathscr{P})$ is metrizable, and every $\operatorname{ANR}(\mathscr{P})$, each point of which is a $G_{\delta}$-set, is metrizable, where $\mathscr{P}$ denotes the class of all paracompact $p$-spaces.
\end{abstract}

1. Introduction. In this paper we shall discuss $\operatorname{ANR}(\mathscr{F})$ spaces for some class $\mathscr{F}$ of topological spaces beyond that of metrizable spaces (that is, $\mathscr{F}$ contains nonmetrizable members).

One of the fundamental problems in the theory of $\operatorname{ANR}(\mathscr{F})$ 's is to find useful criterions to determine whether or not a given space is an $\operatorname{ANR}(\mathscr{F})$. In this aspect we believe that Ščepin's result [7] and its elementary but useful generalization [8] for the class of compact spaces are important. In the preceding note [11] we have extended his former result for the class of $\sigma$-locally compact, paracompact $p$-spaces.

In this note we shall extend these results for the class $\mathscr{P}$ of paracompact p-spaces. (They are precisely the perfect preimages of metric spaces, or equivalently, those spaces which are homeomorphic to a closed subspace of the product of a metric space and a compact space.) Then, the purpose of this paper is to show the following:

THEOREM. Every nonmetrizable ANR(P्P) contains a copy of a Tychonoff cube of uncountable weight.

COROLlaRY 1. Every finite dimensional $\operatorname{ANR}(\mathscr{P})$ is metrizable.

The above corollary gives a positive solution to the problem of Telgársky (see [11, Problem 1]). We also prove

COROLlaRY 2. Every $\operatorname{ANR}(\mathscr{P})$, each point of which is a $G_{\delta}$-set, is metrizable. In particular, every first-countable $\operatorname{ANR}(\mathscr{P})$ is metrizable.

In this paper all maps are assumed to be continuous. For the undefined terminology refer to $[1,3,6]$.

Received by the editors October 16, 1984 and, in revised form, March 1, 1985. This material was presented at the 21 th General Topology Symposium held at Tokyo Gakugei University, Tokyo, on June 5. 1985.

1980 Mathematics Subject Classification. Primary 54C55; Secondary 54B35, 54D18, 54F15, 54F45.

Key words and phrases. ANR, paracompact $p$-space, generalized Peano continuum.

1986 American Mathematical Society $0002-9939 / 86 \$ 1.00+\$ .25$ per page 
The author expresses his thanks to Professor M. Tsuda, the referee, and the editor for their careful reading of an early version of this paper and for their valuable comments. He also would like to express deep appreciation to Professor Telgársky for raising the problem considered here and for his constant attention to this paper $[9,10]$.

2. Proofs of our results. We start with the proof of the main Theorem. Let $X$ be an $\operatorname{ANR}(\mathscr{P})$ which contains no copy of a Tychonoff cube of uncountable weight. Then, it suffices to show that $X$ is metrizable. We can assume [5] that $X$ is a closed subspace of a product space $I^{\tau} \times T$ of a Tychonoff cube $I^{\tau}(\tau=w(X))$ and a metric space $T$. Let $r: U_{0} \rightarrow X$ be a retraction for some cozero-set neighborhood $U_{0}$ of $X$ in $I^{\tau} \times T$. Since $T$ is metrizable, there exists a perfect map $q$ from some strongly zero-dimensional metric space $Y$ onto $T$. Take a cozero-set neighborhood $U^{\prime}$ of $X$ in $I^{\tau} \times T$ such that $X \subset U^{\prime} \subset \bar{U}^{\prime} \subset U_{0}$, where $\bar{U}^{\prime}$ denotes the closure of $U^{\prime}$. Put

$$
\begin{aligned}
& p=1_{I^{r}} \times q, \quad Z_{0}=p^{-1}\left(U_{0}\right), \quad Z=p^{-1}\left(U^{\prime}\right), \\
& f_{0}=r \cdot p \mid Z_{0}: Z_{0} \rightarrow X \text { and } f=f_{0} \mid Z .
\end{aligned}
$$

Let $\left\{\mathscr{U}_{i}\right\}_{i=1}^{\infty}$ be a sequence of disjoint clopen covers of $Y$ which satisfies the condition that $\mathscr{U}_{i+1}$ refines $\mathscr{U}_{i}$ and that the mesh of $U_{i}$ is less than $1 / i$ for every $i$. For each $\mathscr{U} \in \mathscr{U}_{i}$ take a point $s(U)$ of $U$. Then put

$$
L(U)=Z_{0} \cap\left(I^{\tau} \times\{s(U)\}\right) .
$$

Since $Z_{0}$ is a cozero-set, $L(U)$ is a countable union of Tychonoff cubes of weight $\tau$. Therefore, its image $f_{0}(L(U))$ is a Lindelöf $\sigma$-space, since $X$ cannot contain nonmetrizable generalized Peano continua [8]. Hence, by [2, Remark following the proof of Theorem 2] there exist a countable set $\tau(U) \subset \tau$ and a map $f_{U}: \pi_{\tau(U)}\left(Z_{0}\right)$ $\rightarrow X$, where $\pi_{\tau(U)}: I^{\tau} \times Y \rightarrow I^{\tau(U)}$ is a natural projection, which satisfy

(1) $f_{0}(z)=f_{U} \circ \pi_{\tau(U)}(z)$ for every $z \in L(U)$;

(2) $\tau(U) \supset \tau(V)$, where $V$ is the unique element of $\mathscr{U}_{i-1}$ which contains $U$.

Next, for each $i$ we define a metric space $M_{i}$ as

$$
M_{i}=\oplus\left\{I^{\tau(U)}: U \in \mathscr{U}_{i}\right\} .
$$

For each $z=(t, y) \in Z$, where $t \in I^{\tau}, y \in Y$, define

$$
\varphi_{i}(z)=i_{U} \circ \pi_{\tau(U)}(t),
$$

where $i_{U}: I^{\tau(U)} \rightarrow M_{i}$ is a natural inclusion map and $U$ is a unique element of $\mathscr{U}_{i}$ containing $y$. Then, $\varphi_{i}: Z \rightarrow M_{i}$ is a continuous map. We shall now consider another topology in the set $Z$, coarser than the original one, which is defined by declaring

(3) a set $U \subset Z$ is open if for every $x \in U$ there exist a natural number $i$ and an open set $W$ of $M_{i}$ such that $x \in \varphi_{i}^{-1}(W) \subset U$.

From (2) this topology is well defined. The set $Z$ with this topology is denoted by $Z^{\prime}$. Next, we show that

(4) the map $f^{\prime}: Z^{\prime} \rightarrow X$, defined by $f^{\prime}(z)=f(z)$ for $z \in Z^{\prime}$, is continuous. 
Let $W$ be any open set in $X$ and take an arbitrary point $z=(t, y)$ in $f^{\prime-1}(W)=$ $f^{-1}(W)$, where $t \in I^{\tau}, y \in Y$. Then, from (1) we can show without difficulty that

(5) $f_{0}(t, y)=f_{0}\left(t^{\prime}, y\right)$ if $\pi_{\nu}(t)=\pi_{\nu}\left(t^{\prime}\right)$, where $\nu=\bigcup_{i=1}^{\infty} \tau\left(U_{i}\right)$ and $U_{i}$ is the unique element of $\mathscr{U}_{i}$ containing $y$.

Since $f$ is continuous, there exist open sets $G \subset I^{\tau}$ and $U \subset Y$ with

(6) $z \in G \times U \subset \bar{G} \times U \subset f^{-1}(W)$.

Then, for some $i$ there exist open sets $O^{\prime} \subset I^{\tau\left(U_{i}\right)}$ and $H^{\prime} \subset I^{\mu}$, where $\mu=\tau-\nu$, such that

$$
U_{i} \subset U, \quad O=\pi_{\tau\left(U_{i}\right)}^{-1}\left(O^{\prime}\right), \quad H=\pi_{\mu}^{-1}\left(H^{\prime}\right), \quad t \in O \cap H \subset G .
$$

For each $j \geqslant i$ put

$$
K_{j}=\zeta_{i}^{-1}\left(O^{\prime}\right) \cap\left(I^{\tau} \times U_{j}\right) .
$$

Note that $z \in K_{j}$ for each $j \geqslant i$. We show that there exists a $k \geqslant i$ such that

(7) $f\left(K_{k}\right) \subset W$.

On the contrary, assume that there exists a sequence $\left\{z_{j}=\left(t_{j}, y_{j}\right)\right\}_{j \geqslant i}$ of points in $Z$ such that

(8) $z_{j} \in K_{j}$ and $f\left(z_{j}\right) \in X \backslash W$.

Then, taking a subsequence if necessary, we can assume that the sequence $\left\{\pi_{\nu}\left(t_{j}\right)\right\}$ converges to some point $s_{0}$ of the compact metric space $\overline{\pi_{\nu}(G)}$. Let $s$ be an accumulation point of the sequence $\left\{t_{j}\right\}$ in $I^{\tau}$. Note that $\pi_{\nu}(s)=s_{0}$. Put $z_{\infty}=(s, y)$. Then, $z_{\infty} \in \bar{Z} \subset Z_{0}$. Since $f_{0}$ is continuous and $f=f_{0} \mid Z$, we have from (8) that $f_{0}\left(z_{\infty}\right) \in X \backslash W$. On the other hand, put

$$
w=\left(t^{\prime}, y\right), \quad \text { where } \pi_{\nu}\left(t^{\prime}\right)=s_{0} \quad \text { and } \pi_{\mu}\left(t^{\prime}\right)=\pi_{\mu}(t) .
$$

Then, $w \in \bar{G} \times U$, and hence from (5) and (6) we have $f_{0}\left(z_{\infty}\right)=f_{0}(s, y)=f_{0}\left(t^{\prime}, y\right)$ $=f\left(t^{\prime}, y\right) \in W$. This is a contradiction. Hence, (7) holds, and then, (4) follows from (3) and (7). Put

$$
\varphi=\Delta \varphi_{i}: Z \rightarrow \prod_{i=1}^{\infty} M_{i} \quad \text { and } Z^{*}=\varphi(Z) .
$$

Then, $\varphi^{\prime}: Z^{\prime} \rightarrow Z^{*}$, defined by $\varphi^{\prime}(z)=\varphi(z)$ for $z \in Z^{\prime}$, is continuous, and is a quotient map from (3). Note that from (4) $f^{\prime}(z)=f^{\prime}\left(z^{\prime}\right)$ if $\varphi^{\prime}(z)=\varphi^{\prime}\left(z^{\prime}\right)$. Hence, there exists a map $f^{*}: Z^{*} \rightarrow X$ satisfying $f^{\prime}=f^{*} \circ \varphi^{\prime}$. Note that $\varphi: Z \rightarrow Z^{*}$ is the composition of the identity map $i: Z \rightarrow Z^{\prime}$ and the natural quotient map $\varphi^{\prime}$ : $Z^{\prime} \rightarrow Z^{*}$. Hence, $f=f^{*} \circ \varphi$. Put

$$
X^{*}=\varphi\left(p^{-1}(X)\right) \text { and } g=f^{*} \mid X^{*}: X^{*} \rightarrow X .
$$

We shall show that $g$ is a perfect map. For every $x \in X$ we have $g^{-1}(x)=\varphi\left(p^{-1}(x)\right)$. Hence, $g^{-1}(x)$ is compact, since $p$ is perfect. Next, we show that $g$ is a closed map. Let $F$ be any closed set of $X^{*}$. Put

$$
H=\varphi^{-1}(F) \cap p^{-1}(X) .
$$


Then, $g(F)=f^{*} \circ \varphi(H)=f(H)=r \circ p(H)=p(H)$. Hence, $g(F)$ is closed in $X$, since $p$ is perfect and $H$ is closed in $p^{-1}(X)$. Therefore, $X$ is metrizable, since $g$ is perfect and $X^{*}$ is metrizable. That completes the proof of the Theorem.

Proof of THE COROLlaries. Let $X$ be a nonmetrizable ANR( $\mathscr{P})$. Then, by our main theorem, $X$ contains a topological copy of a Tychonoff cube $I^{\omega_{1}}$, where $\omega_{1}$ is the first uncountable cardinal. Therefore, $X$ is infinite dimensional, and each point in $I^{\omega_{1}} \subset X$ is not a $G_{\delta}$-set. That completes the proof of the corollaries.

REMARK. One of the main points of our theorem is that retractions are not necessarily closed in our cases. It seems that the assumption of the existence of closed retraction is a quite restricted one. For example, there are no closed retractions from the Euclidean plane $R^{2}$ onto its $x$-axis $R \times\{0\}$. For the more special case when $X$ is an AR with a closed retraction our results follow from [4, Theorem 1.1] and [12, Corollary 2], respectively.

\section{REFERENCES}

1. R. Engelking, General topology, PWN, Warsaw, 1977.

2. __ On functions defined in Cartesian products, Fund. Math. 59 (1966), 221-231.

3. S. T. Hu, Theory of retracts, Wayne Statc Univ. Press, Detroit, Mich., 1965.

4. B. S. Klebanov, On the metrization of $\varphi$-space, Dokl. Akad. Nauk SSSR 246 (1979), 809-812; English transl., Soviet Math. Dokl. 20 (1979), 557-560.

5. J. Nagata, A note on M-spaces and topologically complete spaces, Proc. Japan Akad. 45 (1969), $541-543$.

6. T. Przymusinski, Collectionwise normality and absolute retracts, Fund. Math. 98 (1977), 61-73.

7. E. V. Ščepin, Every finite-dimensional compact absolute neighborhood retract is metrizable, Dokl. Akad. Nauk SSSR 233 (1977), 304-307; English transl., Soviet Math. Dokl. 18 (1977), 402-406.

8. Sur les applications continues des cubes de Tihonov, C. R. Acad. Sci. Paris Ser. A-B 288 (1979), 257-260.

9. R. Telgársky, A letter to the author on May 31, 1984.

10. A letter to the author on January 3, 1985.

11. K. Tsuda, A note on Ščepin's theorem, Proc. Amer. Math. Soc. 91 (1984), 167-170.

12. Y. Yajima, The normality of $\Sigma$-products and the perfect $\kappa$-normality of Cartesian products, J. Math. Soc. Japan 36 (1984), 689-699.

Department of Mathematics, Ehime University, Matsuyama 790, Japan 